\title{
Tapioca starch films reinforced with microcrystalline cellulose for potential food packaging application
}

\author{
Siti Hajar OTHMAN ${ }^{1,2 *}$, Noorasyikin Abd MAJID ${ }^{1}$, Intan Syafinaz Mohammed Amin TAWAKKAL ${ }^{1}$ (D), \\ Roseliza Kadir BASHA ${ }^{1}$ (D), Norhazirah NORDIN ${ }^{1}$, Ruzanna Ahmad SHAPI'I ${ }^{1}$
}

\begin{abstract}
This work is directed towards developing biodegradable films from biopolymer that is sustainable and environmentally friendly particularly tapioca starch (TPS) films. However, the usage of TPS films for food packaging application has been limited due to the poor mechanical, barrier and thermal properties. Microcrystalline cellulose (MCC) at different concentrations (0 to $10 \mathrm{wt}$.\%) was incorporated as the filler into the films to form TPS/MCC composite films via solvent casting method in order to improve the limited properties of the films. The TPS/MCC films were characterized in terms of physical, mechanical, barrier, and thermal properties. It was found that $3 \mathrm{wt} . \% \mathrm{MCC}$ was the ideal concentration of filler that resulted to the highest performance of the films in terms of mechanical and barrier properties. Thermal properties of the films were also improved with the addition of MCC into the films. In conclusion, TPS/MCC films produced in this study exhibit improved properties and have the potential to be used for food packaging application.
\end{abstract}

Keywords: barrier property; food packaging; mechanical property; microcrystalline cellulose; tapioca starch; thermal property.

Practical Application: Sustainable and environmentally friendly food packaging material.

\section{Introduction}

Nowadays, environmental pollution resulted from the usage of non-degradable materials such as petroleum-based materials for packaging applications is becoming crucial (Risyon et al., 2016). Among the packaging applications, food packaging represents the single largest element whereby demand for food packaging has been growing due to the increase in the number of world population. Nevertheless, this problem can be solved by replacing the non-degradable materials with biodegradable materials such as biopolymer (Othman, 2014; Othman et al., 2017a). Biopolymer is a polymer produced by living organism that consists of monomeric units that are covalently bonded, forming chain like molecules. Biopolymer falls into two categories which are naturals and synthetic biopolymer. The natural biopolymers are more environmental friendly than synthetic biopolymers and among them, starch particularly tapioca starch (TPS) is a favourable biopolymer due to its low cost, widely accessible, exhibiting thermoplastic and biodegradability properties (Almasi et al., 2010). TPS is isotropic, odorless, tasteless, colourless, non-toxic, and biologically degradable (Dufresne et al., 2013). TPS is also edible and thus suitable for food packaging application as it can be consumed and helps to minimize waste.

However, the usage of starch as film material exhibits some drawbacks due to poor mechanical, thermal, and gas barrier properties, and weak water resistances that limit its application particularly in the presence of water and humidity (Yang et al.,
2009; Othman et al., 2017b). Adding filler such as cellulose to the biopolymer films to form composite is a promising way to improve these properties (Angles \& Dufresne, 2000). The polysaccharide structure of starch and cellulose has similar chemical structure which makes it possible to form hydrogen bonds between both components (Kumar \& Singh, 2008; Ma et al., 2008). It will most likely lead to a decent bond of the matrix filler interface without the expansion of a coupling agent or surface modifications starches (Kumar \& Singh, 2008). Microcrystalline cellulose (MCC) has been used as ecofriendly filler and reinforcement for biopolymers (Lu et al., 2005). MCC has no harmful effects on human health and is used as highly effective additive to improve the product and processing properties in various fields of application from foodstuffs, cosmetics and pharmaceuticals to products for the paper and textile industries.

MCC incorporated into TPS films resulted to TPS/MCC composite films with improved properties. Nevertheless, properties of the composite films such as physical, mechanical, barrier and thermal properties are very much dependent on the filler concentration. Starch-based film materials with a variety of improved properties can be tailored by adding different concentration of MCC filler. For example, concentration of filler will affect the morphology of the films produced. Morphology is very much related to the colour and transparency of the films which are important factors that determine product

${ }^{1}$ Department of Process and Food Engineering, Faculty of Engineering, Universiti Putra Malaysia, Serdang, Selangor, Malaysia

${ }^{2}$ Materials Processing and Technology Laboratory, Institute of Advanced Technology, Universiti Putra Malaysia, Serdang, Selangor, Malaysia

*Corresponding author: s.hajar@upm.edu.my 
acceptability by the consumers. Moreover, concentration will also affected affinity between filler and matrix whereby good affinity enhances mechanical and thermal properties of the biopolymer films (Trache et al., 2016). Likewise, concentration of filler determines the formation of tortuous pathway inside the biopolymer matrix thus affecting the barrier properties of the produced composite films. Therefore, ideal concentration of filler in biopolymer matrix is important to be investigated in order to produce efficient packaging films.

In this work, MCC at different concentrations (0-10 wt.\%) were incorporated into TPS film as the filler. The effect of concentrations of MCC on physical properties (morphology, colour, and transparency), mechanical properties (tensile strength (TS), Young's modulus (YM), and elongation at break (EAB)), barrier properties (water vapour permeability (WVP) and oxygen permeability), and thermal properties (glass transition temperature ( $\mathrm{Tg}$ ) and melting temperature (Tm)) of the TPS/MCC films were investigated. Finally, ideal concentration of MCC that could produce the most efficient TPS film for food packaging application was determined.

\section{Materials and methods}

\subsection{Materials}

Materials used in this work include tapioca starch (Thye Huat Chan Sdn Bhd, Thailand), glycerol plasticizer (R\&M Chemicals, UK), microcrystalline cellulose (Sigma-Aldrich Co., Ireland) with average particle size of $20 \mu \mathrm{m}$, calcium chloride (R\&M Chemicals, UK) and magnesium nitrate 6-hydrat (HmbG Chemicals, German).

\subsection{Preparation of films}

Neat TPS films (control) were prepared by solvent casting method. Film forming solution was prepared by mixing $3.0 \mathrm{~g}$ of tapioca starch and $100 \mathrm{~mL}$ of distilled water. Glycerol ( $30 \% \mathrm{w} / \mathrm{w}$ of starch: $0.90 \mathrm{~g}$ ) was added as plasticizer. The solution was heated to $75{ }^{\circ} \mathrm{C}$ under constant stirring using magnetic stirrer (FAVORIT HS0707V2, Indonesia) to obtain starch gelatinization. An amount of $50 \mathrm{~mL}$ of the solution was poured into acrylic petri dish which acted as casting plate and dried in air conditioned room $\left(25^{\circ} \mathrm{C}\right)$ for 48 hours on flat table (to ensure the film thickness was uniform). After dried, the film was peeled off from casting plate and stored in desiccator (Relative humidity (RH): $50 \%$, Temperature: $25^{\circ} \mathrm{C}$ ) prior to analysis.

To prepare the TPS/MCC composite films, MCC was first prepared at different concentrations $(1,3,5$, and $10 \mathrm{wt}$.\% based on starch weight). MCC was dispersed in $100 \mathrm{~mL}$ of distilled water and stirred vigorously for 1 hour using magnetic stirrer. The fully dispersed suspensions were then homogenized using ultrasonic probe (Qsonica Sonicator Q500, USA) for 10 minutes. Then, $0.90 \mathrm{~g}$ of glycerol was added into the suspension and mixed vigorously for 20 minutes using magnetic stirrer. Next, $3.0 \mathrm{~g}$ of starch and $100 \mathrm{~mL}$ distilled water were added slowly into the solution and mixed vigorously at $75^{\circ} \mathrm{C}$ using magnetic stirrer. An amount of $50 \mathrm{~mL}$ of the mixture was casted on acrylic petri dish and dried for 48 hours in air conditioned room. After dried, the film was peeled off from casting plate and stored in desiccator prior to analysis. Every analysis conducted on the films was repeated triplicate to ensure accuracy of the result.

\subsection{Physical properties}

The morphology of TPS/MCC composite films were observed using SEM (Model S-3400N, Hitachi, Japan). All the films were coated with gold prior observing under SEM. The test was carried out with voltage of $15 \mathrm{kV}$. The thickness of the films was measured using a digital vernier caliper (Mannesmann, Germany) at five random positions. The average values of the thickness were used to determine the mechanical and barrier properties of the films.

Colour analysis was conducted using HunterLAB Ultra Scan VIS 8 Spetrophotometer with D65 illuminant (Emam et al., 2014). Colour reading was expressed in terms of CIELAB colour parameters: $\mathrm{L}^{*}=$ Lightness $(0=$ black, $100=$ white $)$; $a^{*}=$ Greenness (-), Redness (+); $b^{*}=$ Blueness (-), Yellowness $(+)$. Measurements were taken at 10 random positions on each of the film. Total colour difference $\left(\Delta \mathrm{E}^{*}\right)$ of the films was determined using the following equation:

$\Delta E=\left(\Delta L^{*}\right)^{2}+\left(\Delta a^{*}\right)^{2}+\left(\Delta b^{*}\right)^{2}$

Transparency of the films was also determined using HunterLAB Ultra Scan VIS 8 Spectrophotometer at visible region (400-700 nm) according to ASTM D1746 (Sothornvit et al., 2009). From the data obtained, transparency at $600 \mathrm{~nm}$ wavelength was determined.

\subsection{Mechanical properties}

Mechanical properties of the films which include TS, YM, and EAB were determined using texture analyzer (TA XT Plus, UK) according to ASTM D882. Five film samples were cut into $100 \mathrm{~mm} \times 15 \mathrm{~mm}$. The samples were then clamped between the grips at initial gauge separation length of $60 \mathrm{~mm}$ and the test was run with crosshead speed of $0.5 \mathrm{~mm} \mathrm{sec}^{-1}$.

\subsection{Barrier properties}

WVP was determined by gravimetric methods using water vapour permeability cup (Yasuda, No.318, Japan) according to JIS Z 0208 method. Anhydrous calcium chloride was used as desiccant with $0 \% \mathrm{RH}$ and was placed inside the cup. The films were cut into circular shapes and sealed to the cup using combination of paraffin and bee wax solution (ratio 4:1). The cups were then placed inside desiccator containing saturated magnesium nitrate solution to provide a constant RH of $50 \%$. The desiccators were placed inside an incubator with the temperature control at $30^{\circ} \mathrm{C}$. The weight of the cup was recorded every 24 hours until the difference in mass was not more than $10 \%$. WVP was calculated as follows:

$W V P=(\Delta m \Delta t A)(L \Delta p)$

where $\Delta \mathrm{m} / \Delta \mathrm{t}$ is moisture gain weight per time $(\mathrm{g} / \mathrm{s}), A$ is exposed surface area of the film $\left(\mathrm{m}^{2}\right), L$ is thickness of the film $(\mathrm{mm})$, and $\Delta \mathrm{p}$ is difference of partial pressure (Jouki et al., 2013). 
Oxygen permeability was determined using oxygen analyzer (Mocon OpTech, USA). Oxygen transmission rate (OTR) of the films was determined for $50 \mathrm{~cm}^{2}$ circular film using an optical measuring system. The test was carried out at room temperature at a pressure of 3 Psi according to ASTM F2714-08 (Souza et al., 2012). The film was put in the chamber and clamped tightly. Pure oxygen was introduced into the upper half of sample chamber while nitrogen gas was injected into the lower half of chamber.

\subsection{Thermal properties}

Thermal properties of the films were investigated using differential scanning calorimetry (DSC) (Mettler Toledo 822, USA). An amount of $5 \mathrm{mg}$ sample film was cut and placed into an aluminium pan and sealed. The film was heated from 30 to $300^{\circ} \mathrm{C}$ with a heating scan rate of $10^{\circ} \mathrm{C} / \mathrm{min}$. Tg and $\mathrm{Tm}$ were then determined from the heating scan.

\section{Results and discussion}

\subsection{Morphology of the films}

The morphology structure of polymer composite is an important characteristic to be investigated because it will ultimately determine the many properties of the polymer composite. Figures 1(a), 1(b), and 1(c) show SEM images of the neat TPS, 3 wt.\% TPS/MCC and 10 wt.\% TPS/MCC films respectively. It can be seen from Figure 1(a) that neat TPS film which act as the control exhibits a relatively smooth morphology. This finding seems consistent to the finding of Wittaya (2009) whereby he also observed smooth surface structure of neat rice starch film viewed using SEM.

Figures 1(b) and (c) illustrate the SEM micrographs of TPS/MCC composite films incorporated with 3 and $10 \mathrm{wt} . \%$ MCC respectively. The smooth morphology of the neat TPS film seem to disappear with the incorporation of MCC into TPS films. Comparing between both figures, Figure 1(b) shows that MCC was dispersed relatively uniform on the surface of the film compared to Figure 1(c) perhaps due to low concentration of MCC added into the film (3 wt.\% compared to $10 \mathrm{wt} . \%$ ). Figure 1(c) shows that dispersion of MCC was not uniform in the TPS matrix and that there was some formation of MCC agglomerates. This was due to high concentration of MCC added into the starch matrix (10 wt.\%) that resulted to the poor dispersion of MCC. The interaction between MCC and TPS matrix became weak when excess amount of MCC was incorporated into the TPS film thus resulted to the formation of agglomerates.
This finding is consistent to the finding of Rico et al. (2016). They found the same trend of result whereby the dispersion of MCC was not uniform in the wheat starch matrix and MCC agglomerates were formed, especially in wheat starch film incorporated with high concentration of MCC. According to Wittaya (2009), distribution of microcrystalline cellulose from palm pressed fiber (MCPF) in the rice starch films was also affected by the MCPF content. He found that addition of MCPF was more or less evenly distributed within the rice starch films. However, at high MCPF concentration (15 to 40\%), some MCPF are not fully individualized and form small aggregates in the rice starch films thus consistent with current finding.

\subsection{Physical properties}

One of the important physical properties of a packaging film is the colour. Colour can influence consumer acceptance towards the film. Appearance of the food product packaged with the film and observed by the consumer can also affect acceptance of the consumer towards the product. Thus, another important property is transparency. Generally food packaging material with high transparency is desirable. Packaging films should exhibit high gloss and high transparency for a good visual presentation of products. Figure 2 shows colour and transmittance results of neat TPS and TPS/MCC composite films.

From Figures 2(a) - (d), it can be seen that addition of MCC into TPS films resulted to a slight decrease in $\mathrm{L}^{*}$ and $\mathrm{a}^{*}$ values but a slight increase in $\mathrm{b}^{*}$ and $\triangle \mathrm{E}$ values. Neat TPS film without addition of MCC filler acted as control sample. It is worth noted that from observation, neat TPS film was generally colourless, translucent and clearer, which can be proved from high $L^{*}$ value of 92.91 . The $L^{*}$ values decreased slightly from 92.91 to 91.99 with addition of $10 \mathrm{wt} . \%$ MCC into the films. According to Wittaya (2009), the decrease in $L^{*}$ value of the films with addition of MCPF was possibly due to the original dark colour of the microcrystalline cellulose. The films became darker as can evidenced by the decrease in $L^{*}$ value with the addition of MCPF. The $\mathrm{L}^{*}$ values were also influenced by MCC concentration added into the TPS films whereby $10 \mathrm{wt} . \%$ MCC concentration gave lower value of lightness compared to $3 \mathrm{wt} . \%$ MCC concentration most probably due to excess MCC content and that MCC particles were not dispersed well in the TPS film. However, the decrement was very slight and almost not significant.

Figure 2(b) shows that $\mathrm{a}^{\star}$ values of the TPS films decreased slightly with the increase in MCC concentration. All $\mathrm{a}^{\star}$ values were negative values, which indicated that the colour of the

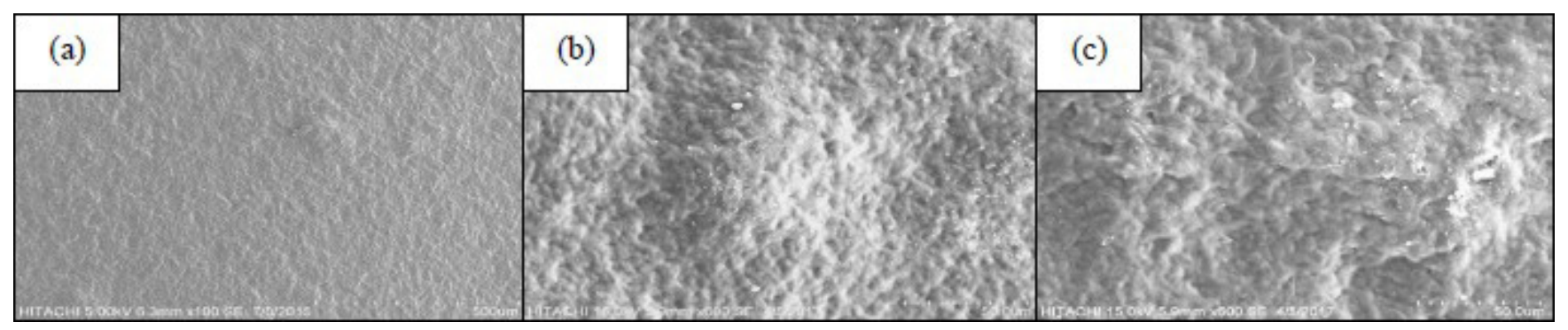

Figure 1. Morphology of (a) neat TPS, (b) 3 wt.\% TPS/MCC, and (c) 10 wt.\% TPS/MCC films. 
(a) $L^{*}$

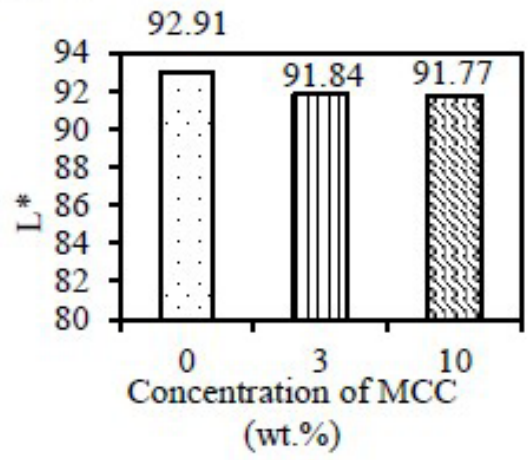

(d) $\Delta \mathrm{E}^{*}$

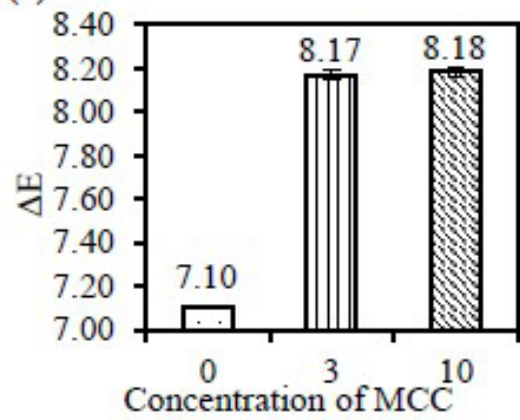

(wt.\%) (b) $a^{*}$

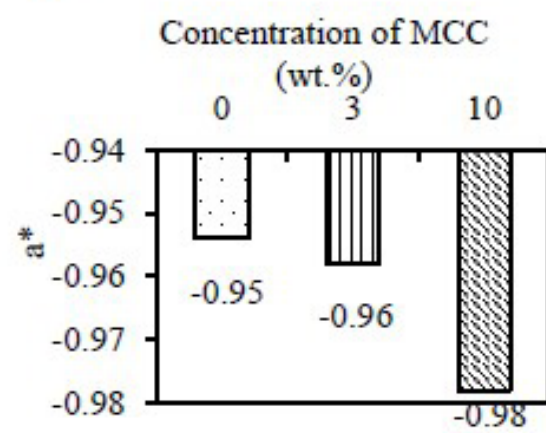

(e) Transmittance (\%) at 400-

$700 \mathrm{~nm}$

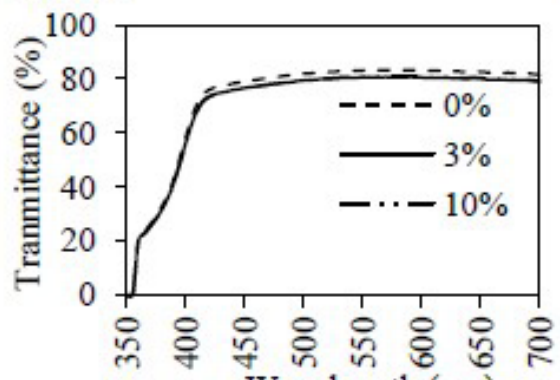

(c) $b^{*}$

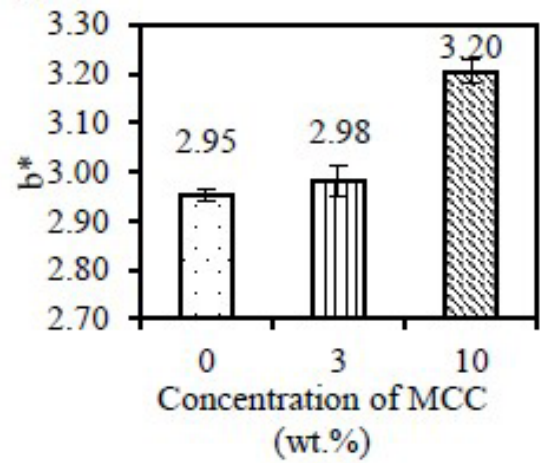

(f) Transmittance (\%) at 600 $\mathrm{nm}$

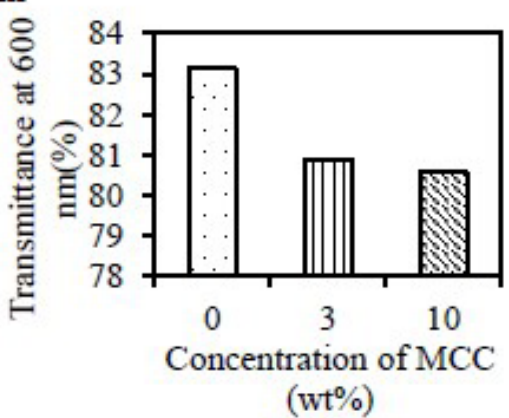

Figure 2. Colour parameters in terms of $(a) L^{*},(b) a^{*},(c) b^{*}$ and (d) $\triangle E$ and transmittance of TPS and TPS/MCC composite films at (e) the spectra of visible region (400-700 nm) and (f) $600 \mathrm{~nm}$.

films were deviated towards green. However, the $\mathrm{a}^{*}$ values were close to zero and thus the green tone could be considered to be almost non-noticeable. Figure 2(c) demonstrates that the films exhibit positive $b^{*}$ values. The $b^{*}$ values seem to increase slightly with the increase in MCC concentration which indicated a shift towards yellow colour. This was due to the increase in solid content of the MCC in the starch film that caused the films to become yellower. The $\Delta \mathrm{E}$ value of the films was also calculated to determine the colour changes in the TPS films with the addition of MCC. Based on Figure 2(d), $\Delta \mathrm{E}$ values did not much differ from each other, but the addition of MCC into the TPS films did show slight increment in $\triangle \mathrm{E}$ values. The $\Delta \mathrm{E}$ values between the films incorporated with 3 and $10 \mathrm{wt} . \%$ of MCC were nearly the same and thus not much difference in terms of colour changes as a whole.

The percentage of light transmittance in the spectra of visible region $(400-700 \mathrm{~nm}$ ) provides the basis of transparency measurement whereby the higher the transmittance, the more transparent is the material. Figure 2(e) clearly shows that neat TPS films exhibited the highest percentage of transmittance in the spectra of the visible region compared to TPS/MCC composite films. Figure 2(f) shows the transmittance value at $600 \mathrm{~nm}$ which reflects the transparency of the films and also provides some information on the particle size and degree of dispersion of the fillers within the matrix (Chen et al., 2009). From Figure 2(f), it can be clearly seen that the transparency of the neat TPS films decreased with the addition of MCC. The transmittance was decreased further with the increase in concentration of MCC.
This was because MCC particles in the starch matrix caused a blocking effect on the virtually transparent TPS film at $600 \mathrm{~nm}$ and hence reduced the transmittance. At high concentration (10wt.\%) of MCC, the agglomeration as can be evidenced from Figure 1(c) further increased the blocking effect on the films due to the large size of the agglomerates. This trend is consistent to the finding of Shapi'i \& Othman (2016) whereby they found that transmittance of TPS films increased with the addition of chitosan and that the transmittance also increased with the increase in chitosan concentration from 20 to $80 \mathrm{wt} . \%$.

Visual appearances of the films were shown in Figure 3. The transparency of the films was compared by observing the images in Figure 3. All of the films produced were transparent as the wordings on the paper could be clearly seen through the films. Based on the observation, neat TPS film was almost fully transparent while the other films became semi-transparent with the addition of MCC. The transparency was seen to reduce with the increase in MCC concentration in the starch matrix. TPS film incorporated with $10 \mathrm{wt} . \%$ MCC exhibited the lowest transparency compared to other TPS/MCC composite films. This was due to MCC particles that were not dispersed well in the film and agglomerated when high amount of MCC was incorporated into the films as proven by SEM image (Figure 1(c)). According to Lee et al. (2008), transparency of films could be affected by the homogeneity and amount of filler. They indicated that the filling of cellulose fiber in starch decreased the transparency of the starch films. 


\subsection{Mechanical properties}

Figures 4(a), (b), and (c) show the TS, EAB, and YM of TPS films and TPS/MCC composite films added with different concentrations of MCC respectively. TS is the maximum tensile stress that can be sustained by the sample film during tension test. $\mathrm{EAB}$ indicates the flexibility and elongation capacity before the film starts to break (Lopez et al., 2014). YM is a measurement of stiffness of the film when tensile force stretches the film vertically. From Figure 4(a), it can be seen that TS of the films increased from 1.67 to $2.35 \mathrm{MPa}$ with the increase in concentration of MCC from 0 to $3 \mathrm{wt} . \%$. This finding indicated that a high compatibility occurred between starch matrix and MCC fillers thus improved TS. However, addition of MCC fillers at high concentration (>3 wt.\%) resulted to reduction of TS of TPS films.

The increase in TS of the TPS films with the addition of 1 and 3 wt.\% MCC was due to the good bonding/interface between the starch matrix and MCC filler resulted from relatively uniform distribution of MCC as can be observed from Figure 1(b). According to Lu et al. (2005), good bonding between starch matrix and cottonseed linter cellulose crystallite filler was due to the three dimensional hydrogen bonds network between different components of starch and cellulose. This finding is consistent to the work of Wittaya (2009) whom found that tensile strength of rice starch film increased with the incorporation of MCPF at ideal concentration.

Meanwhile, the reduction of TS at high concentration of MCC occurred due to a decrease in the compatibility of starch and MCC. Surface of the films that were conglomerated and MCC that did not disperse well in the starch film due to the formation of agglomeration (Figure 1 (c)) led to a decrease in TS. The agglomeration of the films resulted to formation of weak point on the films. The weak point could be broken easily when stress was applied at the point thus decreased TS. The reduction of TS was also due to the excess amount of MCC that led to an increase in intermolecular interaction between MCC that might compete with the interaction between MCC fillers and the starch matrix (Lani, 2014).

Figure 4 (b) shows that the trend of EAB result seems to be reciprocal to the trend of TS results (Figure 4(a)). The EAB values decreased from $23 \%$ to $16 \%$ with the increase in MCC

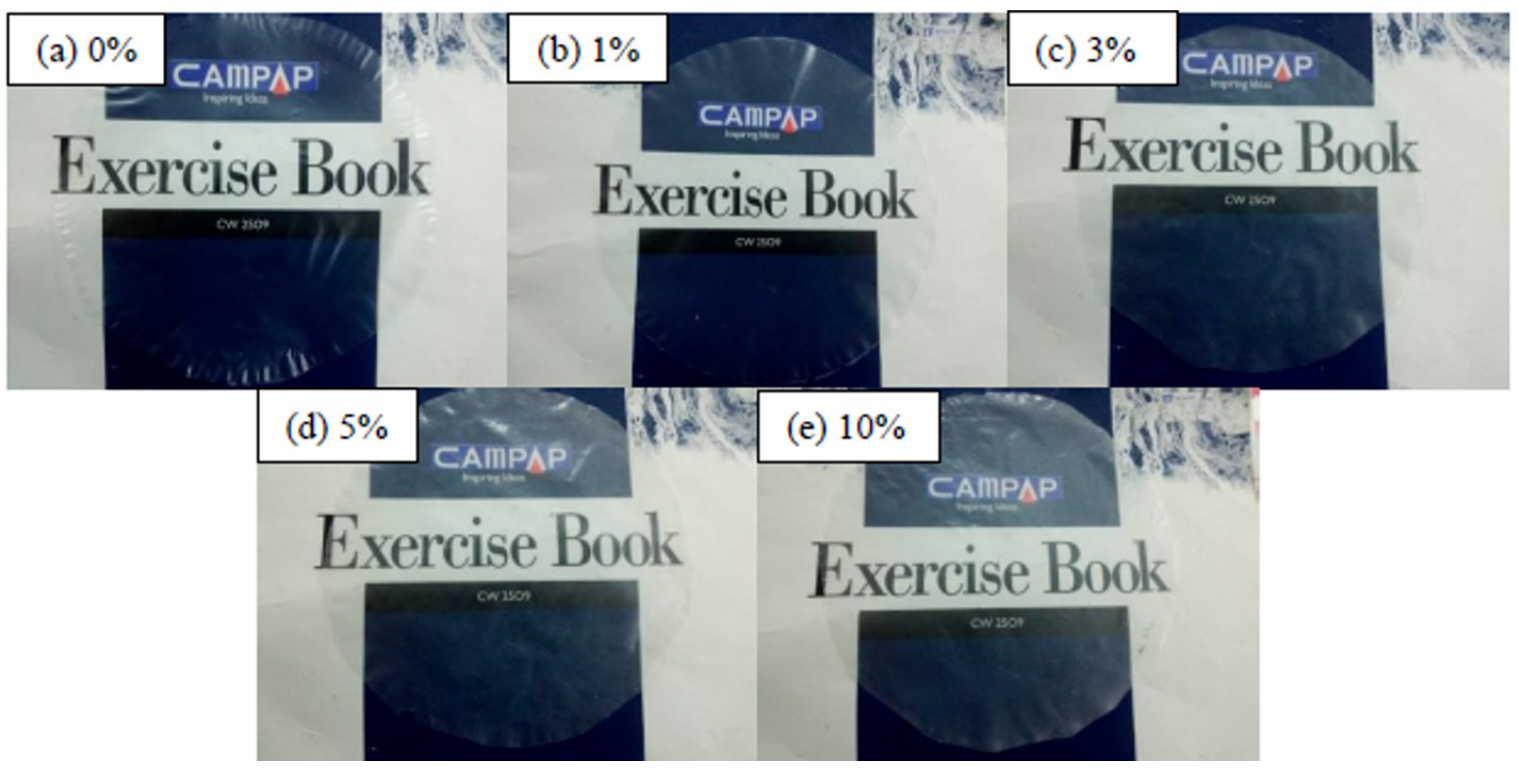

Figure 3. Visual appearance of the TPS and TPS/MCC composite films.

(a) Tensile Strength

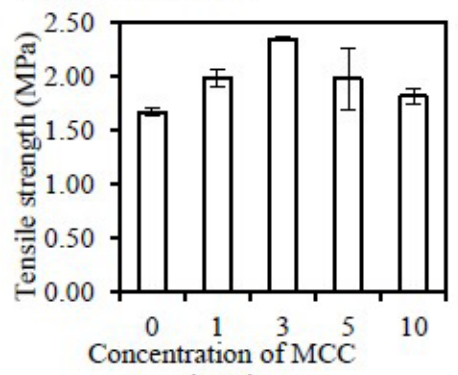

$(\mathrm{wt} \%)$ (b) Elongation at Break

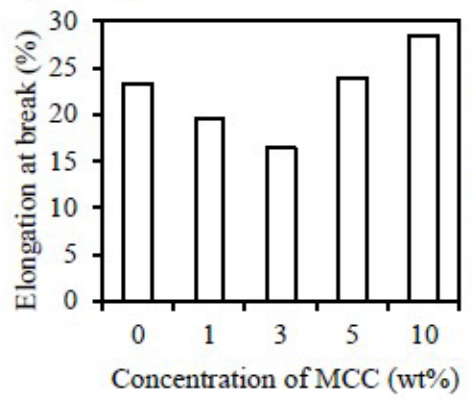

(c) Young's Modulus

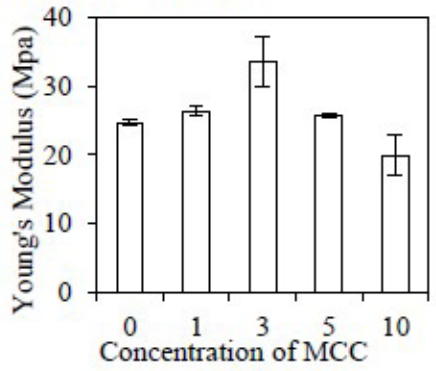

$(\mathrm{wt} \%)$

Figure 4. (a) TS, (b) EAB, and (c) Young's Modulus of TPS films and TPS/MCC composite films. 
concentration from 0 to $3 \mathrm{wt} . \%$. The reduction of EAB shows that the film became less flexible due to the high TS values of the films. The EAB reduced when low concentration of MCC (1 and 3 wt.\%) was added into TPS films because MCC was dispersed well (Figure 1(b) in the film matrix at these range of concentration, thus increased TS and reduced EAB.

The agglomeration of MCC in the film matrix is one of the factors that caused the EAB values to increase from $16 \%$ to $29 \%$ when MCC concentration was increased from 3 to $10 \mathrm{wt} . \%$ as can be evidenced from Figure 1(c). MCC fillers were not homogeneously distributed thus formed small aggregates in the starch film matrix. This was possibly due to the presence of high content of MCC fillers which contributed to retard the intermolecular interaction between starch films and matrix thus increased flexibility. Agglomeration also resulted to the increment in free volume in the film matrix thus promoted flexibility of the films and increased EAB (Ma et al., 2008).

Low YM value indicates that a film exhibits high elasticity (Lee et al., 2008) or low stiffness value. Figure 4(c) shows the effect of different concentrations of MCC on YM of TPS films. Based on Figure 4(c), YM of the neat TPS film was found to be $24.60 \mathrm{MPa}$. TPS film added with $3 \mathrm{wt}$ \% MCC exhibits the highest YM value of $33.61 \mathrm{MPa}$. YM values of the TPS films seem to increase with the increase in concentration of MCC from 0 to 3 wt.\%. The excellent interaction bonds and intermolecular forces between starch and MCC molecules that resulted by good dispersion of MCC in the starch matrix (Figure 1(b)) enhanced the stiffness of the film. High intermolecular forces between the starch and MCC increased films rigidity and TS, hence reduced elasticity and increased stiffness of the film, thus high YM.

When the MCC concentration was further increased to 5 and 10 wt.\%, YM dropped dramatically to 25.65 and $19.88 \mathrm{MPa}$ respectively. The reduction of YM occurred due to the formation of MCC agglomerates in the starch films. MCC at high concentration was not homogeneously dispersed in the starch film thus forming agglomerates that affected the interaction bonds between starch and MCC thus reduced intermolecular forces. The poor intermolecular forces between starch and MCC promoted increment in elasticity and decrement in stiffness of the films, thus low YM.

\subsection{Barrier properties}

Two of the important barrier properties of packaging material include water barrier and oxygen barrier. Water barrier and oxygen barrier properties of packaging material are important aspects for food packaging application in which the properties will affected the shelf life of the product packaged with the material. For example, moisture and oxygen content inside a packaged leads to microorganism growth that can reduce the shelf life of food. Thus, it is important to investigate the water vapor permeability and oxygen permeability of packaging material in order to control moisture and oxygen transfer between the food and the surrounding atmosphere. Figures 5(a) and (b) show the effect of addition of different concentrations of MCC filler on the WVP and OTR of TPS films.

Figure 5(a) shows that WVP decreased from 3.71E-08 to 3.37E-08 $\mathrm{g} \mathrm{m} / \mathrm{kPa}$ day $\mathrm{m}^{2}$ day with the addition of $10 \mathrm{wt} . \%$ MCC into the TPS films. There is a trend of a decrease in WVP with the increase in MCC concentration inside the films. The permeability of the water reduced with the increase in concentration of MCC filler because the permeation process was affected by the presence of impermeable MCC which led to tortuosity in the film matrices (Sanchez-Garcia et al., 2008). Water molecules have to go through the tortuous path in the starch film thus lower the WVP values. According to Bharadwaj (2001), the important factor that can affect the permeability of the film is the aggregation and dispersion of the fillers added into the film matrix (Bharadwaj, 2001). The improvement in the WVP of the films was attributed to the formation of hydrogen bond network of cellulose starch in the film matrix.

Supposedly, WVP reduces at low concentration of filler (3 wt.\%) but increases at high concentration of filler (10 wt.\%). This is due to the fact that filler at low concentration is usually dispersed well in the film matrix (Figure 1(b)) thus forming tortuous path that reduces WVP but filler at high concentration usually resulted to formation of agglomeration (Figure 1(c)) which favours water permeability. At high concentration, the fillers are expected to congregate thus decreased the effective contents of filler and facilitated the water vapour permeation. However, in this study, it can be speculated that at high concentration of MCC (10 wt.\%), the hydrophilic properties of the cellulose (a) WVP

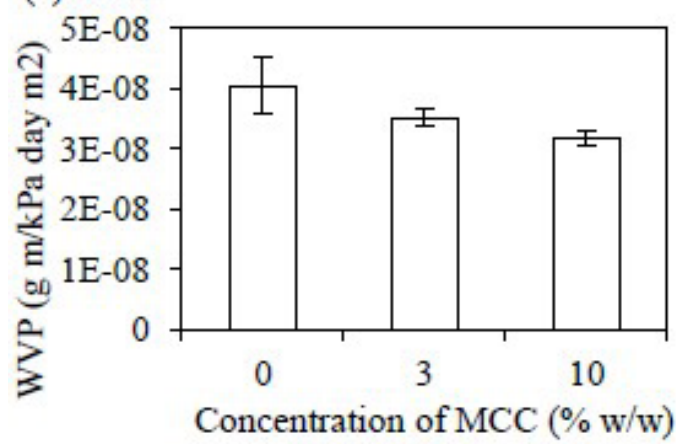

(b) OTR

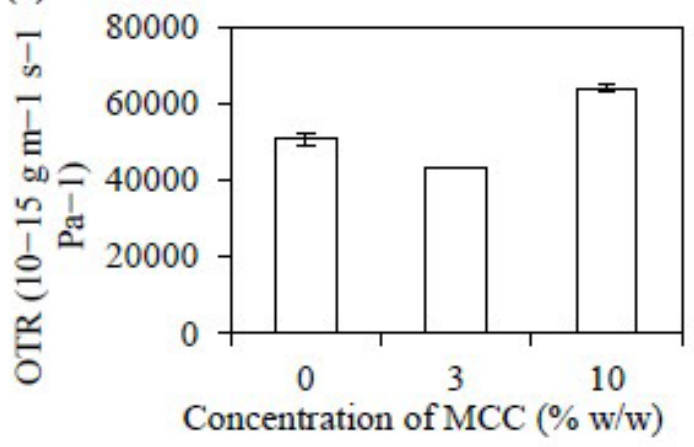

Figure 5. The (a) WVP and (b) OTR of TPS and TPS/MCC composite films. 
which favoured water permeability was playing more significant role compared to the effect of agglomeration. The finding in this work seems to be supported by the work of Ma et al. (2008) whereby the water vapour permeability of thermoplastic pea starch decreased with the increase in MCC filler concentration from 0 to $12 \mathrm{wt} . \%$.

The amount of oxygen gas that, over a given period of time at a steady rate, passes through a substrate is measured by OTR. The films for food packaging application must exhibit low OTR to avoid or reduce oxidative process of the food product during the transport, handling and storage thus increases shelf life (Miller \& Krochta, 1997).

Figure 5(b) shows that addition of 3 wt.\% MCC in TPS films decreased the OTR value of the films due to the well dispersed MCC in the matrix film (Figure 1(b)). MCC created tortuous path that slowed down the transmission of oxygen through the films hence low OTR. When impermeable MCC was incorporated into starch matrix, gas molecules were forced to diffuse around the starch in a random walk due to the MCC rather than taking a straight line path that lies perpendicular to the film surface, hence lower the OTR. However, the incorporation of $10 \mathrm{wt} . \% \mathrm{MCC}$ into the TPS films resulted to the highest OTR value which resulted from the agglomeration of MCC at high concentration and the poor dispersion of MCC in the film (Figure 1(c)). The permeation of gas molecules was probably favoured in the area between the separated phases that caused by agglomeration. Diffusion of the oxygen gas through the films was found to be very much related to the orientation of the filler in the matrix and the state of aggregation and dispersion of these fillers (Bharadwaj, 2001). Improvement in gas barrier properties was due to the concept of the tortuous path of the material.

This finding seems to be consistent to the work of Savadekar \& Mhaske (2012), whereby the oxygen transmission rate of thermoplastic starch soluble film reduced with the increase in the cellulose content inside the film. Starch has high oxygen permeability but the decrease in OTR of nanocomposite films was due to the existence of dispersed nanocellulose fibers which generated a tortuous path for the permeation of oxygen molecules inside the film.

\subsection{Thermal analysis}

Three characteristics of thermal transitions may exist for semicrystalline polymers such as starch which include glass transition for the amorphous composition, melting transition for the crystalline composition and transition due to crystallization. Table 1 tabulates the Tg and Tm of the neat TPS film and TPS film incorporated with $3 \mathrm{wt}$ \% MCC. Note that no crystallization temperature (Tc) data was obtained from DSC for both films due to no occurrence of transition process from amorphous to crystalline stage. Table 1 shows that Tg of neat TPS film

Table 1. Tg and Tm of neat TPS and TPS films incorporated with 3 wt.\% MCC.

\begin{tabular}{cccc}
\hline Films & $\mathrm{T}_{\mathrm{g}}\left({ }^{\circ} \mathrm{C}\right)$ & $\mathrm{T}_{\mathrm{m}}\left({ }^{\circ} \mathrm{C}\right)$ & $\Delta \mathrm{H}(\mathrm{J} / \mathrm{g})$ \\
\hline $\mathbf{0 \%}$ MCC & 39.53 & 320.15 & 121.65 \\
3\% MCC & 44.97 & 322.76 & 173.58 \\
\hline
\end{tabular}

was $39.53{ }^{\circ} \mathrm{C}$ which was consistent to the range of initial $\mathrm{Tg}$ ( 42.42 to $26.96^{\circ} \mathrm{C}$ ) of cassava starch found by Mali et al. (2006) when they varied the concentration of glycerol from 20 to $40 \%$.

Generally, Tg and Tm of TPS film incorporated with $3 \mathrm{wt} . \%$ MCC were higher than that of the neat TPS film. Tg increased from 39.53 to $44.97^{\circ} \mathrm{C}$ while $\mathrm{Tm}$ increased from 320.15 to $322.76^{\circ} \mathrm{C}$ with the addition of $3 \mathrm{wt} . \%$ MCC into TPS films. Tg is associated with changes in the amorphous region of PLA from hard, glassy, and brittle to soft, rubbery, and flexible properties while Tm determines the transition in the crystalline regions from solid to liquid state. Tg and Tm of TPS films increased with the addition of MCC due to the strong and rigid properties of the films resulted from the formation of intermolecular hydrogen bond between MCC and TPS. The intermolecular bond requires higher energy to change from glassy to rubbery state and from solid to liquid state hence higher $\mathrm{Tg}$ and $\mathrm{Tm}$ compared to neat TPS film.

The $\mathrm{Tg}$ decreased due to the decrement of entanglements and interactions of starch polymer chains due to the MCC inclusion. According to Almasi et al. (2010), the enhancement in $\mathrm{Tm}$ indicated that the cellulose content favoured the formation of larger crystal domains and thus lower the mobility of the biopolymer chains to change from solid to liquid state. Kumar \& Singh (2008) also reported the increase in Tm of starch matrix with the incorporation of cellulose. They claimed that the increase in Tm was attributed to anti-plasticization effect of amylopectin rich domains by the incorporation of cellulose microfibers. Angles \& Dufresne (2000) revealed that the strong affinity of amylopectin molecules towards surface of cellulose fiber through high density of hydroxyl groups reduced the global mobility of amylopectin domains and hence high energy was required to change the film from solid to liquid state.

\section{Conclusion}

Concentration of MCC is an imperative factor that affected the dispersion of the MCC fillers in the TPS films. State of dispersion of MCC greatly influences the physical, mechanical, barrier, and thermal properties of TPS films, hence final application of the films as potential food packaging material. This factor needs to be controlled to ensure the efficiency of the film as an alternative food packaging material. It was found that TPS films incorporated with 3 wt.\% MCC has the potential to be used for food packaging application in order to reduce the usage of non-degradable material films due to the improved mechanical, barrier, and thermal properties.

\section{Acknowledgements}

This work was financially supported by the Fundamental Research Grant Scheme (FRGS), Ministry of Higher Education, Malaysia (Project no. 03-01-16-1873FR, Vote no. 5524980).

\section{References}

Almasi, H., Ghanbarzadeh, B., \& Entezami, A. A. (2010). Physicochemical properties of starch-CMC-nanoclay biodegradable films. International Journal of Biological Macromolecules, 46(1), 1-5. http://dx.doi. org/10.1016/j.ijbiomac.2009.10.001. PMid:19828115. 
Angles, M. N., \& Dufresne, A. (2000). Plasticized starch/tunicin whiskers nanocomposites. 1. Structural analysis. Macromolecules, 33(22), 8344-8353. http://dx.doi.org/10.1021/ma0008701.

Bharadwaj, R. K. (2001). Modeling the barrier properties of polymerlayered silicate nanocomposites. Macromolecules, 34(26), 9189-9192. http://dx.doi.org/10.1021/ma010780b.

Chen, Y., Liu, C., Chang, P. R., Anderson, D. P., \& Huneault, M. A. (2009). Pea starch-based composite films with pea hull fibers and pea hull fiber-derived nanowhiskers. Polymer Engineering and Science, 49(2), 369-378. http://dx.doi.org/10.1002/pen.21290.

Dufresne, A., Thomas, S., \& Pothan, L. A. (2013). Biopolymer nanocomposites: processing, properties, and applications. New Jersey: John Wiley \& Sons. http://dx.doi.org/10.1002/9781118609958.

Emam, H. E., Mowafi, S., Mashaly, H. M., \& Rehan, M. (2014). Production of antibacterial colored viscose fibers using in situ prepared spherical Ag nanoparticles. Carbohydrate Polymers, 110, 148-155. http://dx.doi. org/10.1016/j.carbpol.2014.03.082. PMid:24906741.

Jouki, M., Yazdi, F. T., Mortazavi, S. A., \& Koocheki, A. (2013). Physical, barrier and antioxidant properties of a novel plasticized edible film from quince seed mucilage. International Journal of Biological Macromolecules, 62, 500-507. http://dx.doi.org/10.1016/j. ijbiomac.2013.09.031. PMid:24076197.

Kumar, A. P., \& Singh, R. P. (2008). Biocomposites of cellulose reinforced starch: Improvement of properties by photo-induced crosslinking. Bioresource Technology, 99(18), 8803-8809. http://dx.doi.org/10.1016/j. biortech.2008.04.045. PMid:18504125.

Lani, N. S. (2014). Isolation and characterization of nanocellulose from empty fruit bunch fiber for nanocomposite application (Thesis Master). Universiti Teknologi Malaysia, Malaysia. http://dx.doi. org/10.1155/2014/702538.

Lee, D. S., Yam, K. L., \& Piergiovanni, L. (2008). Food packaging science and technology. New York: CRC Press. http://dx.doi. org/10.1201/9781439894071.

Lopez, O. V., Garcia, M. A., Villar, M. A., Gentili, A., Rodriguez, M. S., \& Albertengo, L. (2014). Thermo-compression of biodegradable thermoplastic corn starch films containing chitin and chitosan. Lebensmittel-Wissenschaft + Technologie, 57(1), 106-115. http:// dx.doi.org/10.1016/j.lwt.2014.01.024.

Lu, Y., Weng, L., \& Cao, X. (2005). Biocomposites of plasticized starch reinforced with cellulose crystallines from cottonseed linter. Macromolecular Bioscience, 5(11), 1101-1107. http://dx.doi. org/10.1002/mabi.200500094. PMid:16245266.

Ma, X., Chang, P. R., \& Yu, J. (2008). Properties of biodegradable thermoplastic pea starch/carboxymethyl cellulose and pea starch/ microcrystalline cellulose composites. Carbohydrate Polymers, 72(3), 369-375. http://dx.doi.org/10.1016/j.carbpol.2007.09.002.

Mali, S., Grossmann, M. V. E., García, M. A., Martino, M. N., \& Zaritzky, N. E. (2006). Effects of controlled storage on thermal, mechanical and barrier properties of plasticized films from different starch sources. Journal of Food Engineering, 75(4), 453-460. http://dx.doi. org/10.1016/j.jfoodeng.2005.04.031.

Miller, K. S., \& Krochta, J. M. (1997). Oxygen and aroma barrier properties of edible films: a review. Trends in Food Science \& Technology, 8(7), 228-237. http://dx.doi.org/10.1016/S0924-2244(97)01051-0.

Othman, S. H. (2014). Bio-nanocomposite materials for food packaging applications: Types of biopolymer and nano-sized filler. Agriculture and
Agricultural Science Procedia, 2, 296-303. http://dx.doi.org/10.1016/j. aaspro.2014.11.042.

Othman, S. H., Edwal, S. A. M., Risyon, N. P., Basha, R. K., \& Talib, R. A. (2017a). Water sorption and water permeability properties of edible film made from potato peel waste. Food Science and Technology (Campinas), 37(Suppl. 1), 63-70. http://dx.doi.org/10.1590/1678457x.30216.

Othman, S. H., Hassan, N., Talib, R. A., Basha, R. K., \& Risyon, N. P. (2017b). Mechanical and thermal properties of PLA/halloysite bionanocomposite films: Effect of halloysite nanoclay concentration and addition of glycerol. Journal of Polymer Engineering, 37(4), 381389. http://dx.doi.org/10.1515/polyeng-2016-0062.

Rico, M., Rodríguez-Llamazares, S., Barral, L., Bouza, R., \& Montero, B. (2016). Processing and characterization of polyols plasticizedstarch reinforced with microcrystalline cellulose. Carbohydrate Polymers, 149, 83-93. http://dx.doi.org/10.1016/j.carbpol.2016.04.087. PMid:27261733.

Risyon, N. P., Othman, S. H., Basha, R. K., \& Talib, R. A. (2016). Effect of halloysite nanoclay concentration and addition of glycerol on mechanical properties of bionanocomposite films. Polymers \& Polymer Composites, 24(9), 793-800. http://dx.doi. org/10.1177/096739111602400917.

Sanchez-Garcia, M. D., Gimenez, E., \& Lagaron, J. M. (2008). Morphology and barrier properties of nanobiocomposites of poly(3hydroxybutyrate) and layered silicates. Journal of Applied Polymer Science, 108(5), 2787-2801. http://dx.doi.org/10.1002/app.27622.

Savadekar, N. R., \& Mhaske, S. T. (2012). Synthesis of nano cellulose fibers and effect on thermoplastics starch based film. Carbohydrate Polymers, 89(1), 146-151. http://dx.doi.org/10.1016/j.carbpol.2012.02.063. PMid:24750616.

Shapi'i, R. A., \& Othman, S. H. (2016). Effect of concentration of chitosan on the mechanical, morphological and optical properties of tapioca starch film. International Food Research Journal, 23(Suppl.), S187-S193.

Sothornvit, R., Rhim, J. W., \& Hong, S. I. (2009). Effect of nano-clay type on the physical and antimicrobial properties of whey protein isolate/clay composite films. Journal of Food Engineering, 91(3), 468-473. http://dx.doi.org/10.1016/j.jfoodeng.2008.09.026.

Souza, A. C., Benze, R., Ferrão, E. S., Ditchfield, C., Coelho, A. C. V., \& Tadini, C. C. (2012). Cassava starch biodegradable films: Influence of glycerol and clay nanoparticles content on tensile and barrier properties and glass transition temperature. LebensmittelWissenschaft + Technologie, 46(1), 110-117. http://dx.doi.org/10.1016/j. lwt.2011.10.018.

Trache, D., Hussin, M. H., Hui Chuin, C. T., Sabar, S., Fazita, M. R., Taiwo, O. F., Hassan, T. M., \& Haafiz, M. K. (2016). Microcrystalline cellulose: Isolation, characterization and bio-composites application - A Review. International Journal of Biological Macromolecules, 93(Pt. A), 789-804. http://dx.doi.org/10.1016/j.ijbiomac.2016.09.056. PMid:27645920.

Wittaya, T. (2009). Microcomposites of rice starch film reinforced with microcrystalline. International Food Research Journal, 16, 493-500.

Yang, L., Liang, G., Zhang, Z., He, S., \& Wang, J. (2009). Sodium alginate/ Na-rectorite composite films: Preparation, characterization, and properties. Journal of Applied Polymer Science, 114(2), 1235-1240. http://dx.doi.org/10.1002/app.30521. 